Over $66 \%$ of patients had a radiologically inserted gastrostomy (RIG) tube and remainder a percutaneous endoscopic gastrostomy (PEG) tube. Patients had PEG placement if they had normal respiratory function (overnight oximetry, vital capacity and no NIV). Placement was unsuccessful in 3 patients (RIG 2; PEG 1); 2 had jejunostomy and one (RIG) declined further intervention. Five patients had complications; two (RIG) had pneumoperitoneum (managed conservatively); two had chest infections (RIG 1, PEG 1) and one had a site infection.

30-day (\%) mortality as follows: no tube placed, 39; patient declined, 30; too unwell, 66; not yet indicated, 0. 1-year mortality (\%) as follows: no tube placed, 74; patient declined, 70; too unwell, 78; not yet indicated, 25 .

Overall mortality (\%) in tube placement as follows: 30-day, 11; 1-year, 57 (RIG, 30-days, 10; 1-year, 68; PEG, 30-days, 13; 1-year, 43). *

Abstract PWE-195 Table 1 showing mortality by year

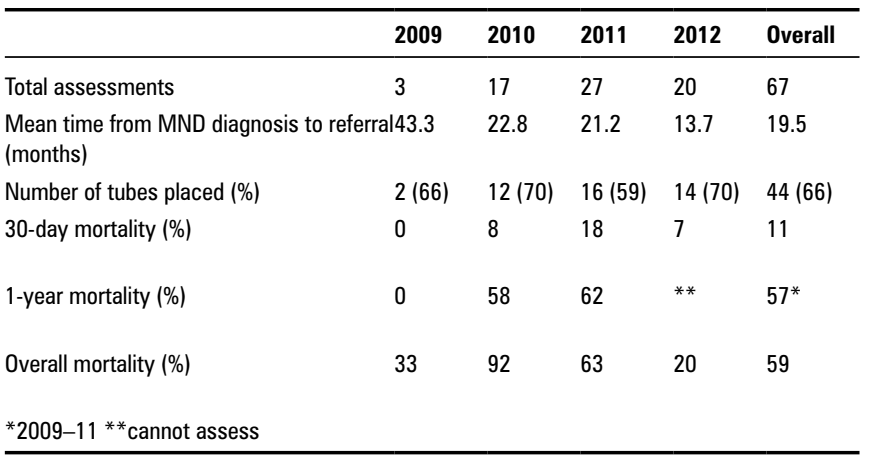

Conclusion Our results show high short-term mortality in all patients referred for nutritional assessment with MND, demonstrating the advanced stage of disease as nutritional status deteriorates. Mortality is lower in the PEG group; a reflection of less respiratory comorbidity or disease stage?

Patients are being referred at earlier stages in their disease; appropriately identifying patients who benefit may provide better outcomes.

Disclosure of Interest None Declared.

\section{PWE-196 PREVALENCE OF OBESITY, BY SPECIALTY, AMONGST INPATIENTS IN THE SOUTH OF ENGLAND}

doi:10.1136/gutjnl-2013-304907.484

1. ${ }^{*} \mathrm{~T}$ Ambrose, ${ }^{2} \mathrm{~S}$ Cullen, ${ }^{2} \mathrm{G}$ Baker, ${ }^{3} \mathrm{M}$ Smith, ${ }^{4} \mathrm{M}$ Elia, ${ }^{5} \mathrm{R}$ Leach, ${ }^{1} \mathrm{~A}$ De Silva. ${ }^{1} \mathrm{G} a s t r o-$

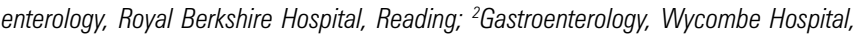
High Wycombe; ${ }^{3}$ Gastroenterology, St Thomas' Hospital, London; " 4 nstitute of Human Nutrition, Southampton; ${ }^{5}$ Respiratory Medicine, St Thomas' Hospital, London, UK

Introduction Rates of obesity, defined by Body Mass Index (BMI) $\geq 30 \mathrm{~kg} / \mathrm{m}^{2}$, are rising in the United Kingdom. The prevalence, by specialty, of obesity amongst inpatients has not been well studied. Methods A multicentre, hospital-wide audit across three Trusts in the South of England was performed on a single day to ascertain the prevalence of obese inpatients by specialty. Patients were classified according to specialty (medical, surgical, orthopaedic, intensive care) and allocated a BMI category based on National Institute for Clinical Excellence guidelines (1).

Results 1163 patients were audited (575 male). 778 were medical patients, 222 surgical, 130 orthopaedic, and 33 intensive care.

Mean age was 69.3 years with a significant mean age difference between specialties (medical 71.4, surgical 67.7, orthopaedic 61.9, intensive care 60.4) and independent of the hospital studied.

260 patients $(22 \%)$ had a $B M I \geq 30 \mathrm{~kg} / \mathrm{m}^{2}$, of which $103(9 \%)$ had a $\mathrm{BMI} \geq 35 \mathrm{~kg} / \mathrm{m}^{2}$. A significant difference in this latter group was observed between specialties (orthopaedic 18\%, intensive care $9 \%$, surgical 9\%, medical $7 \%(p<0.001))$.
No effect of gender was observed but there was an independent effect of increasing age and higher BMI.

Conclusion Approximately one quarter of inpatients in our multicentre audit were obese according to BMI criteria with $9 \%$ significantly obese $\left(\mathrm{BMI} \geq 35 \mathrm{~kg} / \mathrm{m}^{2}\right)$. Rates of obesity are similar to rates of malnutrition in hospitals. Higher BMI were observed in orthopaedic and intensive care specialties than in medical and surgical. Doctors, nurses and managers should be aware of this variation for training and resource allocation purposes.

Disclosure of Interest None Declared.

\section{REFERENCE}

1. Obesity: the prevention, identification, assessment and management of overweight and obesity in adults and children. National Institute for Health and Clinical Excellence. December 2006.

\section{Radiology}

\section{PWE-197 IS MRCP A USEFUL INVESTIGATION WHERE THE BILIARY TREE IS NORMAL ON PREVIOUS IMAGING?}

doi:10.1136/gutjnl-2013-304907.485

'B Vadhwana, ${ }^{1} \mathrm{~J}$ Graby, ${ }^{1, *} \mathrm{~A}$ Yusuf, ${ }^{2} \mathrm{~A}$ Sergot, ${ }^{2} \mathrm{C}$ Ramsey, ${ }^{1} \mathrm{~K}$ Monahan. ${ }^{1}$ Gastroenterology: ${ }^{2}$ Radiology, West Middlesex University Hospital, Isleworth, London, UK

Introduction Magnetic Resonance Cholangiopancreatography (MRCP) is increasingly used in the diagnosis of biliary disease, especially in stone disease. It has a high sensitivity and specificity[1] [2]; however its role in the absence of dilated biliary tree on previous imaging is not clear. The aim of this study was to determine the diagnostic yield of MRCP in patients with an undilated biliary tree.

Methods We performed a retrospective observational study of MRCP studies $(\mathrm{n}=119)$ performed between October 2011 and September 2012 at West Middlesex University Hospital using electronic medical records. All MRCPs were reported by a consultant radiologist. MRCP findings were correlated with the presence of dilated (but otherwise normal) or undilated biliary tree on initial imaging (USS/CT), jaundice (bilirubin $>21 \mu \mathrm{mol} / \mathrm{L}$ ) and abdominal pain. Demographics including age and gender were noted. Fisher's exact test was used to analyse binary variables and student's T test for continuous variables using the STATA12 statistical software

Results In patients with a normal biliary tree on previous imaging the yield of MRCP was low with only 2/44 demonstrating stone or other pathology $(p=0.0002)$. Patient referred for MRCP without biliary tree dilatation had a median age 12 years younger $(p=0.033)$ and the indication was more likely to be pain $(p=0.017)$ but not jaundice $(p=1)$ and referrals were not gender related $(p=0.23)$.

Conclusion Our study demonstrates a low diagnostic yield of MRCP in the absence of dilated biliary system on previous USS/CT. Furthermore, the presence of jaundice or abdominal pain does not help to select patients who may benefit from further biliary imaging with MRCP. Routine MRCP in patients with an undilated biliary tree on USS/CT does not appear to be indicated.

Disclosure of Interest None Declared.

\section{REFERENCE}

1. ShanmugamV, Beattie GC, Yule SR, et al. Is magnetic resonance cholangiopancreatography the new gold standard in biliary imaging? British Journal of Radiology (2005) 78, 888-893.

2. Williams EJ, Green J, Bechingham I, et al.Guidelines on the management of common bile ductstones (CBDS). Gut 2008; 57:1004-1021. 